\title{
ELECTROPHRENIC RESPIRATION. II. ITS USE IN MAN ${ }^{2}$

\author{
By JAMES L. WHITTENBERGER, STANLEY J. SARNOFF, AND
} ESTHER HARDENBERGH
}

(From the Department of Physiology, Harvard School of Public Health, Boston)

(Received for publication August 6, 1948)

In a previous investigation it was shown that artificial respiration could be effectively administered to the cat, dog, monkey and rabbit in the absence of spontaneous respiration by electrical stimulation of one or both phrenic nerves $(1,2)$. During the development of the applications of the technique, an opportunity occurred for testing the method in man. A patient with severe chronic, diaphragmatic flutter was operated on for the purpose of 1) crushing the left phrenic nerve and 2) temporarily blocking the right phrenic with procaine. At the time of operation a single, slender, multiplestrand, plastic-covered wire was spirally wound around the right phrenic nerve in its exposed portion and the other end of the lead wire was brought out through a simple needle puncture wound about $2 \mathrm{~cm}$. lateral to the site of incision. The operation was performed under local anesthesia. Five hours later, electrophrenic respiration was begun.

The method is described in detail in a previous communication (2). Briefly, it is the following. An ECG plate secured at the wrist functions as an indifferent electrode for the single stimulating electrode on the phrenic nerve. The stimulating current is derived from a Grass stimulator set for a frequency of $40 / \mathrm{sec}$. and an impulse duration of 2 milliseconds. The current is run through a rotating potentiometer which rhythmically varies the voltage in such a way as to cause the diaphragm to perform a gradual contraction and relaxation similar to that which it performs during spontaneous breathing.

Air flow patterns were obtained with the pneumotachograph developed by Silverman (3) and Silverman and Whittenberger (4). From the pneumotachogram one is able to derive information related not only to the rate and contour of the respiratory pattern but also precise values for the patient's ventilation volume. The level of arterial oxygen saturation was observed throughout the period of phrenic nerve stimulation by means of the Millikan oximeter (5).

The experiments on this patient were designed to achieve five objectives:

1. To ascertain whether electrophrenic respiration could maintain minute volumes equal to or

1 Aided by a grant from The National Foundation for Infantile Paralysis. greater than the spontaneous resting minute volume with submaximal stimulation of one phrenic nerve.

2. To ascertain the ease with which a smooth diaphragmatic motion can be obtained in man.

3. To find out if the unanesthetized patient would cease spontaneous respiratory activity when adequate aeration was supplied by means of phrenic stimulation (as was previously found to be the case in the experimental animal under anesthesia).

4. To estimate the amount of pain or discomfort produced by effective electrical stimulation of the phrenic nerve.

5. To ascertain whether adequate arterial oxygen saturation could be maintained with the submaximal stimulation of one phrenic nerve.

\section{RESULTS}

1. Figure 1 shows the pneumotachograms of $A$ ) spontaneous respiration and $B$ ) electrophrenic respiration. Table I compares values obtained

TABLE I

Respiratory measurements during spontaneous breathing and electrophrenic respiration

\begin{tabular}{l|c|c}
\hline \hline & $\begin{array}{c}\text { Spon- } \\
\text { taneous } \\
\text { respira- } \\
\text { tion }\end{array}$ & $\begin{array}{c}\text { Electro- } \\
\text { phrenic } \\
\text { respira- } \\
\text { tion }\end{array}$ \\
\hline Rate per minute & 26.6 & 23.3 \\
Minute volume in liters & 5.18 & 7.52 \\
Tidal volume in liters & 0.195 & 0.323 \\
Average flow rate during inspiration, in & 12.0 & 15.9 \\
liters per minute & & 47.3 \\
Ratio of time of inspiration to time of total \\
respiratory cycle, in per cent
\end{tabular}

during spontaneous respiration and electrophrenic respiration for respiratory rate, minute and tidal volumes, average flow rate during inspiration, and the fraction of the respiratory cycle taken by the inspiratory phase. These results were obtained at a rate of artificial respiration slightly slower than the patient's spontaneous rate and with submaximal stimulation of one phrenic nerve. It can be 
seen that an exchange of air significantly larger than the patient's spontaneous exchange is readily achieved at a slower than the spontaneous rate. A still larger exchange of air resulted from maximal stimulation. It was established in this patient, as in the experimental animal, that the depth of respiration is proportional to the peak voltage applied. The regularity of the tracing in Figure $\mathrm{I} B$ indicates that the patient was not contributing to the respiratory effort. This phenomenon will be discussed later.

2. It is apparent from Figure $1 B$ that the diaphragmatic contraction produced by electrical stimulation of the phrenic nerve in this patient was a smooth motion. (The minor irregularities during diaphragmatic relaxation in the record are due to the diaphragmatic flutter.) A smooth inspiratory curve was, in fact, predicted from the theoretical basis on which electrophrenic respiration is based; namely, that increasingly forceful contraction of the diaphragm results from the spread of current to include more and more fibers of the phrenic nerve as the voltage is increased. Since the phrenic nerve in man is a single trunk and is considerably larger than that of the experimental animal, a graded voltage spread is more easily and smoothly obtained. As observed visually, the movement of the right diaphragm resulting from phrenic nerve stimulation closely resembled that seen during spontaneous respiration.

3. It was apparent from simple observation of the patient that during artificial respiration there was no respiratory effort other than that of the right diaphragm,. which resulted from electrical stimulation. In order to obtain graphic evidence of this fact, two additional maneuvers were carried out. Figure 2 is a pneumotachogram of spontaneous respiration interrupted by the onset of electrical artificial respiration. There is no evidence that spontaneous respiratory activity occurred after phrenic nerve stimulation had been started. Spontaneous activity would be evident as irregularities in the pattern. The reverse sequence was employed to find out whether, upon abruptly terminating artificial respiration, the lack of spontaneous respiratory activity would be apparent from the lack of air flow as registered on the pneumotachogram. Figure 3 is the result. Artificial respiration was stopped at the signal and it can be seen that no respiratory activity was present until the apnea had persisted for over 16 seconds. Breathing then returned with a gradual increase in amplitude until it regained its pre-stimulus contours. This interesting observation, namely, that
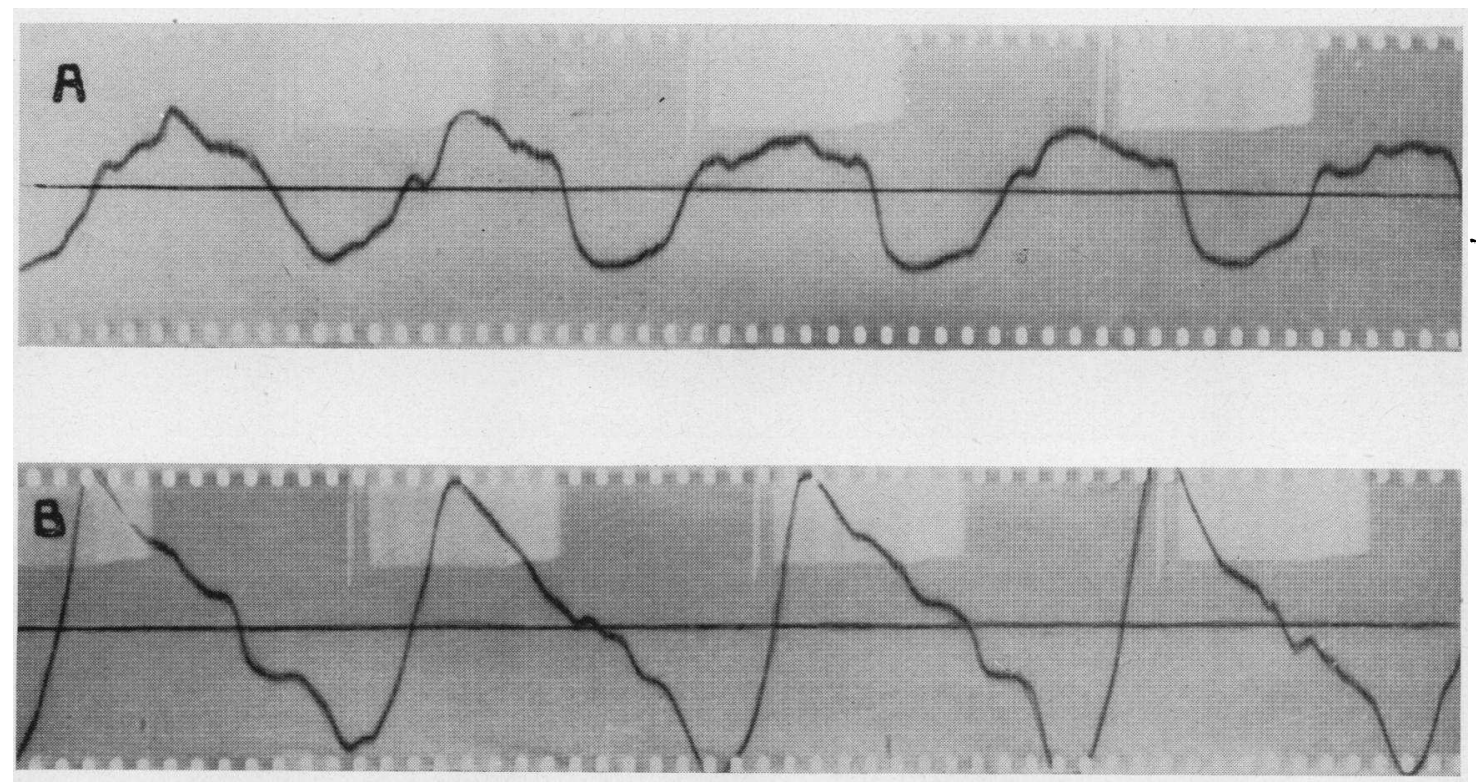

Fig. 1. $A$, Pneumotachogram of Spontaneous Respiration; $B$, Pneumotachogram during ElectroPHRENIC RESPIRATION

Area under the base line represents volume of air flow during inspiration; that above the base line represents expiration. The size of the area is directly proportional to the volume of air exchanged. 

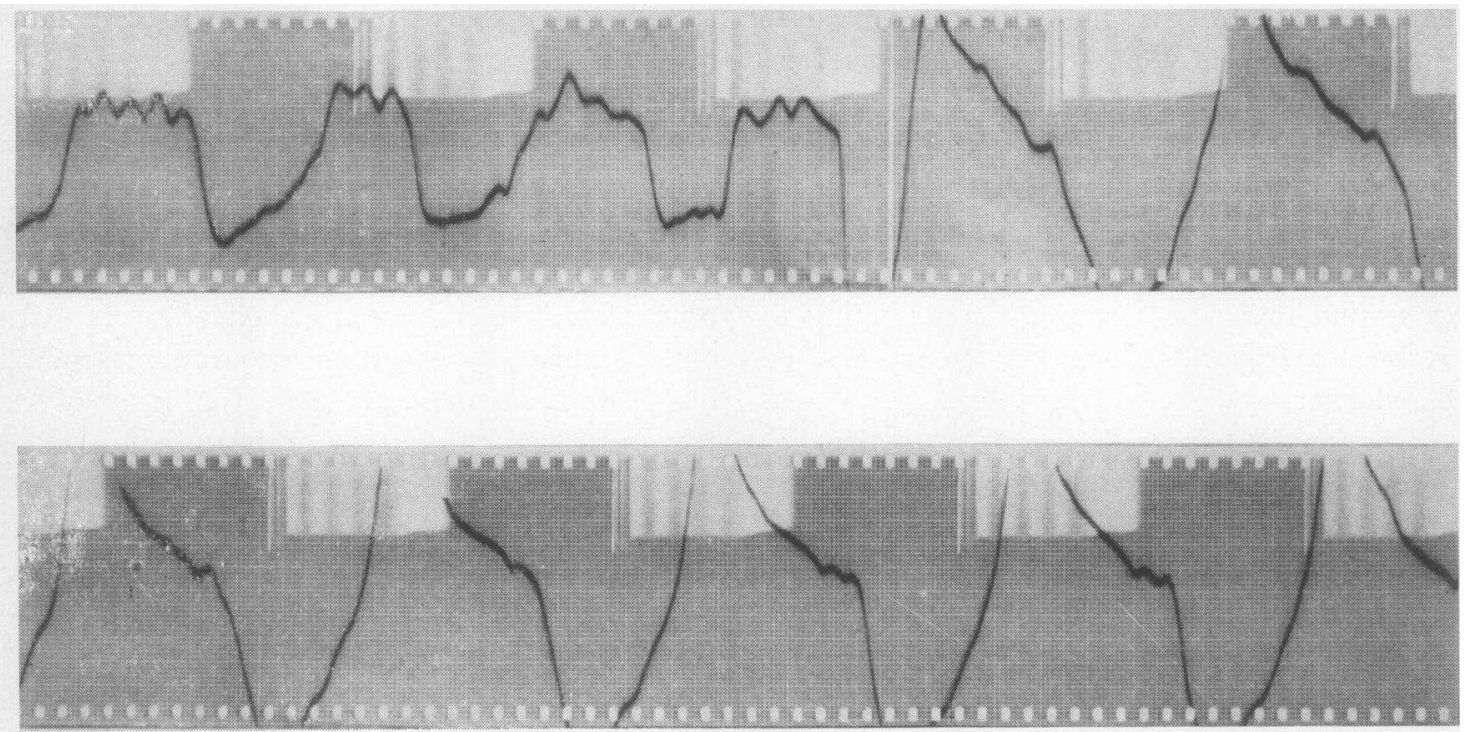

Fig. 2. Pneumotachogram of Spontaneous Respiration Interrupted by the Onset of ElectroPHRENIC RESPIRATION

Phrenic nerve stimulation was started at the signal. The two strips are continuous.
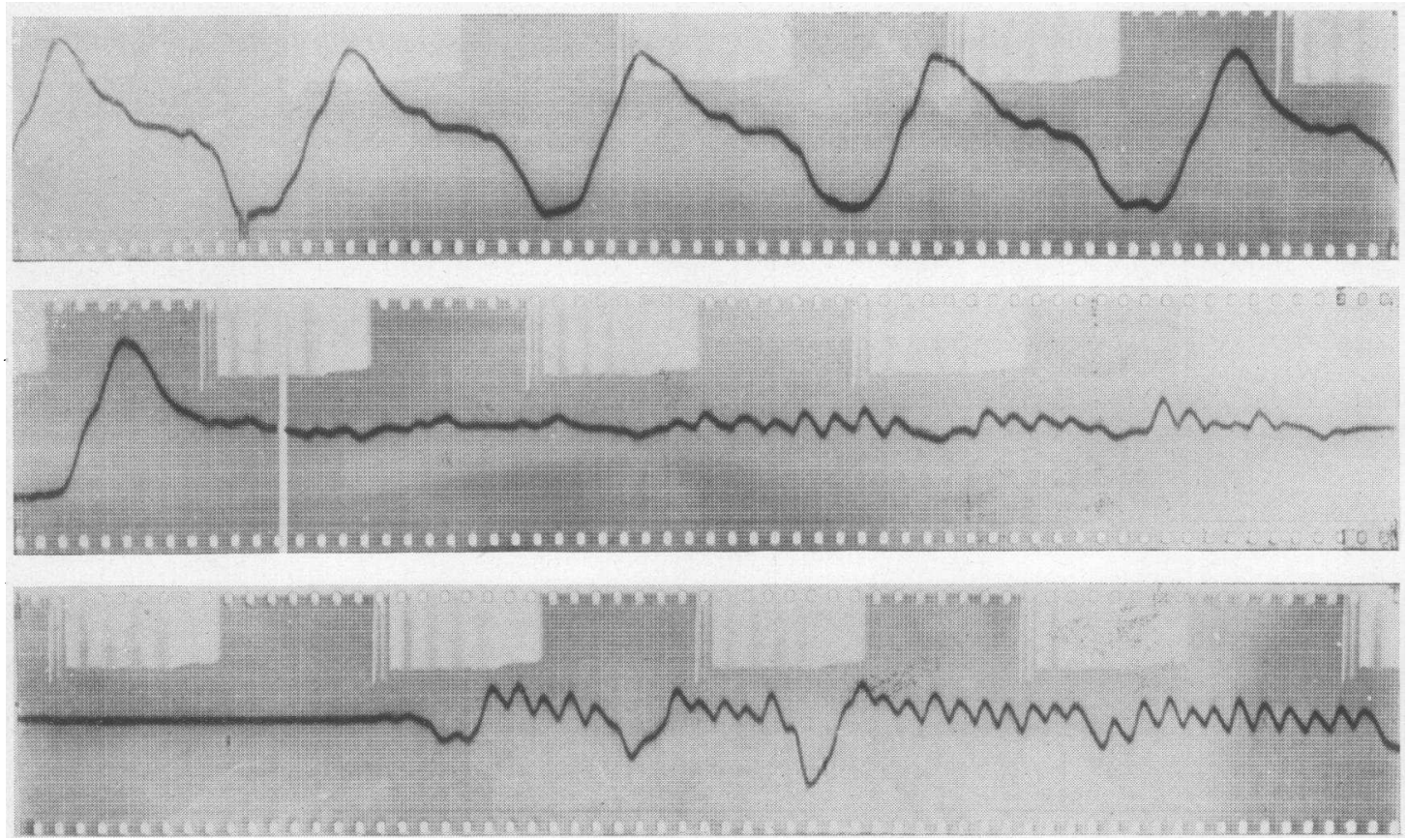

Fig. 3. Apnea Induced by Electrophrenic Respiration

The patient was on artificial respiration at the start of this pneumotachogram. At the signal, phrenic stimulation was discontinued and the tracing shows the absence of spontaneous respiratory effort during the period immediately following cessation of artificial respiration. The minor irregularities are due to the diaphragmatic flutter. The three strips are continuous. 
the individual completely relinquishes spontaneous control of respiration while on electrophrenic respiration, is not yet fully explained. Animal experiments in which blood gas tensions were observed indicate that the prompt cessation of spontaneous respiration is not due to a fall in carbon dioxide brought about by overventilation. The neural pathways involved in this phenomenon are being investigated.

4. The evaluation of pain in this patient was not easy. During phrenic nerve stimulation the patient experienced discomfort referred to the right shoulder along the ridge of the trapezius muscle. This information was obtained only on direct questioning, was never volunteered. The patient did not appear to be in pain and fell asleep several times during the longer periods of artificial respiration. Conclusions as to the degree and importance of the intensity of the discomfort produced will have to be deferred until a variety of patients have been studied.

5. It was thought, prior to our experience with this patient, that the relatively stable mediastinum of the human subject might prevent the adequate aeration of the contralateral lung when only one phrenic nerve was stimulated, even though reasonable respiratory minute volumes were achieved. In order to obtain evidence in this matter, arterial oxygen saturation (by the oximeter method) was observed during spontaneous respiration and during artificial respiration in the absence of spon-

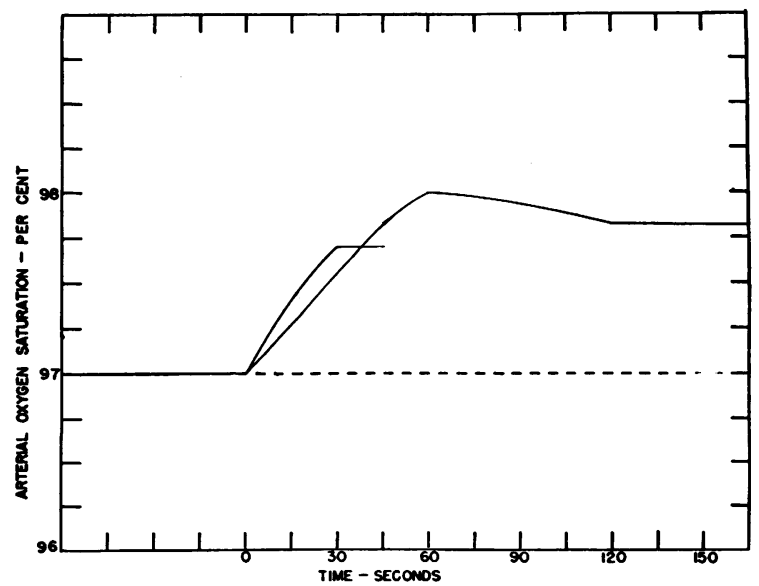

Fig. 4. Effect of Electrophrenic Respiration on Arterial Oxygen Saturation in the Absence of Spontaneous Respiratory Activity

The patient breathed room air throughout. taneous respiratory activity. Figure 4 shows the results. It is clear that a small but definite rise in arterial saturation occurred within 60 seconds of the onset of electrophrenic respiration. A rise of 1 per cent in saturation at the upper range of the oxyhemoglobin dissociation curve indicates a rise of $10 \mathrm{~mm}$. $\mathrm{Hg}$ or more in the mean effective alveolar partial pressure of oxygen. This signifies an improvement in lung ventilation out of proportion to a 1 per cent rise in oxygen saturation. The oximeter was calibrated by having the patient inhale 99.6 per cent oxygen.

Artificial respiration by phrenic nerve stimulation was induced in this patient for several periods varying in length from five to 76 minutes on two occasions during the five days following electrode application. The procedure apparently resulted in no functional damage to the nerve, since fluoroscopic examination of spontaneous diaphragmatic activity on the day of electrode removal revealed excursions equal in amplitude to those observed before the experiment.

\section{CONCLUSIONS}

1. Artificial respiration by phrenic nerve stimulation can be performed in man.

2. A smooth, gradual, diaphragmatic contraction occurs when an increasing voltage is applied to the phrenic nerve. The diaphragm thus performs a motion closely resembling that which it performs during natural inspiration.

3. Respiratory minute volumes in excess of the patient's spontaneous minute volumes can readily be obtained with the submaximal stimulation of one phrenic nerve.

4. The depth of respiration is proportional to the peak voltage applied to the phrenic nerve in man as in the experimental animal.

5. Adequate oxygenation of the blood can be maintained by electrophrenic respiration in the absence of spontaneous respiration.

6. The human subject, like the experimental animal, completely relinquishes spontaneous control of respiration when electrophrenic respiration is induced.

\section{ACKNOWLEDGMENTS}

The authors wish to express their thanks to Doctors K. Emerson, J. L. Blodgett, and Lewis Dexter for the opportunity of studying this patient. 


\section{BIBLIOGRAPHY}

1. Sarnoff, S. J., Hardenbergh, E., and Whittenberger, J. L., Electrophrenic respiration. Science, 1948, $108,482$.

2. Sarnoff, S. J., Hardenbergh, E., and Whittenberger, J. L., Electrophrenic respiration. Am. J. Physiol., in press.

3. Silverman, L., Respiratory air flow characteristics and their relation to certain lung conditions occurring in industry. J. Indust. Hyg. \& Toxicol., 1946, 28, 183.

4. Silverman, L., and Whittenberger, J. L. In preparation.

5. Millikan, G. A., The oximeter, an instrument for measuring continuously the oxygen saturation of arterial blood in man. Rev. Scient. Instruments, 1942, 13, 434. 